\title{
Genome-Wide RNAi Screen Identifies Regulators of Cardiomyocyte Necrosis
}

\author{
Zhaokang Cheng, ${ }^{* \dagger}{ }^{\dagger}$ Matthew Combs, ${ }^{\ddagger}$ Qiang Zhu, ${ }^{\ddagger}$ Peng Xia, ${ }^{\dagger}$ Zachary Opheim, ${ }^{\ddagger}$ Joel Parker, ${ }^{\S} \|$ \\ Christopher P. Mack, ${ }^{\ddagger}, \perp$ and Joan M. Taylor ${ }^{*}, \pm, \perp$ \\ ${ }^{\dagger}$ Department of Pharmaceutical Sciences, Washington State University, Spokane, Washington 99210, United States \\ ${ }^{\ddagger}$ Department of Pathology, ${ }^{\S}$ Department of Genetics, "Lineberger Cancer Center, and ${ }^{\perp}$ McAllister Heart Institute, University of \\ North Carolina, Chapel Hill, North Carolina 27599, United States
}

Supporting Information

\begin{abstract}
Regulation of cellular death is central to nearly all physiological routines and is dysregulated in virtually all diseases. Cell death occurs by two major processes, necrosis which culminates in a pervasive inflammatory response and apoptosis which is largely immunologically inert. As necrosis has long been considered an accidental, unregulated form of cellular death that occurred in response to a harsh

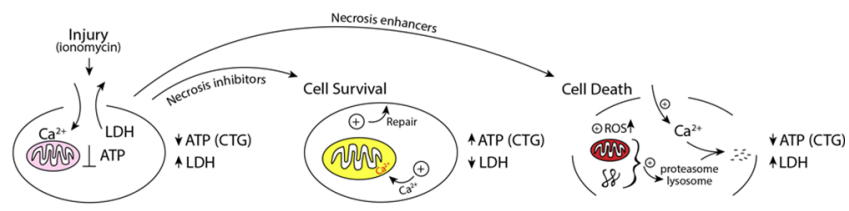
environmental stimulus, it was largely ignored as a clinical target. However, recent elegant studies suggest that certain forms of necrosis can be reprogrammed. However, scant little is known about the molecules and pathways that orchestrate calciumoverload-induced necrosis, a main mediator of ischemia/reperfusion (IR)-induced cardiomyocyte cell death. To rectify this critical gap in our knowledge, we performed a novel genome-wide siRNA screen to identify modulators of calcium-induced necrosis in human muscle cells. Our screen identified multiple molecular circuitries that either enhance or inhibit this process, including lysosomal calcium channel TPCN1, mitophagy mediatorTOMM7, Ran-binding protein RanBP9, Histone deacetylase HDAC2, chemokine CCL11, and the Arp2/3 complex regulator glia maturation factor- $\gamma$ (GMFG). Notably, a number of druggable enzymes were identified, including the proteasome $\beta 5$ subunit (encoded by PSMB5 gene), which controls the proteasomal chymotrypsin-like peptidase activity. Such findings open up the possibility for the discovery of pharmacological interventions that could provide therapeutic benefits to patients affected by myriad disorders characterized by excessive (or too little) necrotic cell loss, including but not limited to IR injury in the heart and kidney, chronic neurodegenerative disorders, muscular dystrophies, sepsis, and cancers.
\end{abstract}

KEYWORDS: cardiac, ischemia/reperfusion, programmed necrosis, proteasome, autophagy

$\mathrm{H}$ eart disease is the leading cause of morbidity and mortality in the developed world. Nearly 1.5 million people experience an acute myocardial infarction (MI) each year in the United States alone, and up to $1 / 3$ of survivors continue to suffer from ischemic cardiomyopathy. Cardiac ischemia is also pervasive in adult patients particularly women and/or those with underlying congenital heart diseases that afflict up to $1 \%$ of live births. Cardiac ischemia leads to oxygen and nutrient deprivation and stimulates both apoptotic and necrotic cardiomyocyte death (which occur at roughly equal levels following MI). Because cardiomyocytes have very limited regenerative potential, ischemic cell death inevitably reduces heart function and leads to heart failure. The fact that the pathological progression of disease is markedly accelerated by the inflammatory responses prompted by myocyte necrosis highlights the importance of discovering the underlying mechanisms. ${ }^{1,2}$

Cell death occurs by two major processes, apoptosis and necrosis. Apoptosis is a highly regulated, programmed process that is controlled by proteolytic caspases, is dependent on ATP, and is largely immunologically inert. The absence of an inflammatory response is due to the piecemeal fashion by which cell contents are cleared wherein membrane blebbing leads to the progressive release and clearing of encapsulated apoptotic bodies by macrophages while the plasma membrane remains intact. Conversely, necrotic cell death involves loss of plasma membrane integrity, osmotic-pressure-induced cell and organelle swelling, ATP depletion, and ultimately plasma membrane rupture. Necrotic release of cellular contents (including factors that contain so-called damage-associated molecular patterns) culminates in a pervasive inflammatory response. As necrosis has long been considered an accidental, unregulated form of cellular death that occurred in response to a harsh environmental stimulus (i.e., a dramatic change in osmotic pressure, $\mathrm{pH}$, or temperature), it was largely ignored as a clinical target. However, recent elegant studies have uncovered signaling pathways that govern certain forms of necrosis. ${ }^{3}$ Such findings open up the possibility for the discovery of future pharmacological interventions that could

Special Issue: Innovation and Discovery in Cardiovascular Biology

Received: July 18, 2019

Published: September 4, 2019 
provide therapeutic benefits to patients affected by myriad disorders characterized by excessive (or too little) necrotic cell loss, including but not limited to IR injury in the heart.

The most well-characterized necrotic pathway described to date is a process coined "necroptosis" which is induced by the tumor necrosis factor (TNF) family of cytokines. A series of elegant studies revealed that $\mathrm{TNF} \alpha$ treatment induces necrosis by promoting assembly of a multiprotein complex containing receptor-interacting protein kinase 1 (RIPK1), RIPK3, and mixed lineage kinase domain-like (MLKL), while TNF $\alpha$ promotes apoptosis by bridging RIPK1 to other intermediates. $^{4-7}$ During necrosis, phosphorylation of MLKL by RIPK3 induces translocation of MLKL to the plasma membrane, wherein it forms a plasma membrane pore and results in the influx of calcium and sodium ions. This osmotic instability ultimately leads to plasma membrane rupture by incompletely understood processes that are largely independent of mitochondrial dysfunction. Importantly, pharmacological intervention upstream of MLKL translocation (and calcium influx) attenuated TNF-induced necrotic death. Such results provide important proof of concept that enzymatic regulators of necroptosis could be useful therapeutic targets for inflammatory-mediated cell death.

However, the main triggers of ischemia-induced necrotic cell death are oxidative stress and calcium overload, and these insults induce a distinct form of regulated necrosis that is associated with cyclophilin D dependent mitochondrial permeability transition (MPT). ${ }^{8,9}$ While MPT-associated necrosis has been linked to myriad cardiovascular, musculoskeletal, and neural diseases ${ }^{8-12}$ and studies suggest that molecules that function to regulate sarcolemmal- ${ }^{10}$ or mitochondrial-stability ${ }^{13,14}$ are critical regulators of IRmediated necrosis, scant little is known about the specific molecules and pathways that orchestrate these processes. To rectify this critical gap in our knowledge, we recently performed a novel genome-wide siRNA screen to identify modulators of calcium-induced necrosis in human muscle cells. Our screen revealed several critical and novel cellular processes and signaling pathways involved in protecting cells from necrosis. In addition, we also identified the ubiquitinproteasome pathway as essential executors of calcium-overload-induced necrosis in human cardiomyocytes and provided support for the possibility that targeting the proteasome might provide a therapeutic avenue for limiting cardiac injury. Overall, this knowledge provides a rich resource to better understand necrosis and could facilitate the development of novel therapies for heart disease and other degenerative disorders.

\section{METHODS}

Cell Culture. Human bronchial smooth muscle cells (HuBrSMCs, CC-2576, Lonza) were maintained in SmGM-2 Smooth Muscle Growth Medium-2 (CC-3182, Lonza). Transfection with siRNAs was performed with DharmaFECT 1 transfection reagent in SmGM-2 media without gentamycin to reduce cytotoxicity as per manufacturer's instructions. Human adult cardiomyocytes (HCMs, 36044-15, Celprogen) were maintained in Human Cardiomyocyte Complete Media with Serum (M36044-15S, Celprogen). Transfection with siRNAs was performed with Lipofectamine RNAiMAX Transfection Reagent in Human Cardiomyocyte Complete Media. Neonatal rat cardiomyocytes (NRCMs) were isolated from 2 to 4 day old Wistar rats using the Neonatal
Cardiomyocyte Isolation System (Worthington Biochemical Corporation, NJ) and seeded in Media 199 supplemented with $15 \%$ fetal bovine serum (FBS) and 1\% penicillin/streptomycin as described previously. ${ }^{15}$ At $24 \mathrm{~h}$, the culture media was switched to serum-free Media 199 followed by siRNA transfection using HiPerfect transfection reagent (Qiagen) or treatment with indicated chemicals. The siRNA sequences used were as follows: siPSMB5: CAUGGUGUAUCAGUACAAA $[\mathrm{dT}][\mathrm{dT}]$; siPSMA6: UACAAAUAUGGCUAUGAGA$[\mathrm{dT}][\mathrm{dT}]$; siControl: UAAGGCUAUGAAGAGAUAC $[\mathrm{dT}]$ $[\mathrm{dT}]$. The following drug concentrations were used: ionomycin $(0.5 \mu \mathrm{M})$, bortezomib $(1 \mu \mathrm{M})$.

siRNA Screens and Statistical Analysis. All screens were performed with automated liquid handling and plate preparation using the Beckman Coulter Biomek FXP assay workstation and the BioTek MicroFlo Select reagent dispensers. Cell viability data were recorded with BMG Labtech Pherastar FS high-throughput screening plate reader.

The primary siRNA screen was performed with the Dharmacon human genomic siRNA library (72 700 siRNAs targeting 18175 genes). Human bronchial smooth cells (HuBrSMCs, passage 10, 700 cells/well) were transfected with pooled siRNAs $(25 \mathrm{nM})$ using DharmaFECT 1 transfection reagent $(0.05 \mu \mathrm{L} /$ well, Dharmacon $)$ in 384-well clear-bottomed white plates (Greiner). Cells were incubated at $37{ }^{\circ} \mathrm{C} / 5 \% \mathrm{CO}_{2}$ for $96 \mathrm{~h}$ and then treated with ionomycin $(5$ $\mu \mathrm{M}$, Cayman Chemical) for $4 \mathrm{~h}$ to induce necrosis. Cell viability, which has been effectively used as a measure of necrosis in a previous siRNA screen, ${ }^{16}$ was determined by measuring intracellular ATP levels using CellTiter-Glo luminescent assay reagent (Promega) according to the manufacturer's protocol. Nontargeting siRNAs and human UBB siRNAs (Dharmacon) which served as negative and positive controls for siRNA transfection, respectively, were present in every plate. The UBB gene encodes ubiquitin, which targets cellular proteins for degradation by the $26 \mathrm{~S}$ proteasome. Cells lacking ubiquitin are universally lethal; thus, UBB siRNA is routinely used in RNAi screens to monitor transfection efficiency. Treatment with ionomycin or vehicle control were each performed in triplicate.

The secondary screen was conducted against the top 200 ranked genes (100 positive and 100 negative regulator of necrosis) using 4 individual siRNAs for each gene. A hit was verified if at least 2 out of 4 siRNAs targeting a single gene altered cell viability by $\geq 30 \%$ and had a $p$-value $<0.05$ versus nontargeting-siRNA-transfected wells.

For both the primary and secondary screens, count data corresponding to siRNA screen targets were normalized to counts from nontargeted control wells by plate and row of the plate. Normalized estimates were log-transformed and tested for differential abundance using the Significance Analysis for Microarray ${ }^{17}$ algorithm implemented in $R$ package samr. The SAM two-class unpaired test was performed, and screen candidates were evaluated based on the SAM empirical $q$-value and the fold change of count between experimental conditions. Screen candidates were excluded if counts in the experimental control samples were less than the 99th percentile of counts from UBB control wells.

Hits from the secondary screen were selected for a tertiary siRNA screen in human cardiomyocytes (Celprogen) according to the following criteria: (1) At least 2 of the 4 individual siRNAs per gene agreed in effect directionality. (2) Positive regulators of necrosis had a $\log _{2}$ (fold change) greater than 1.2. 

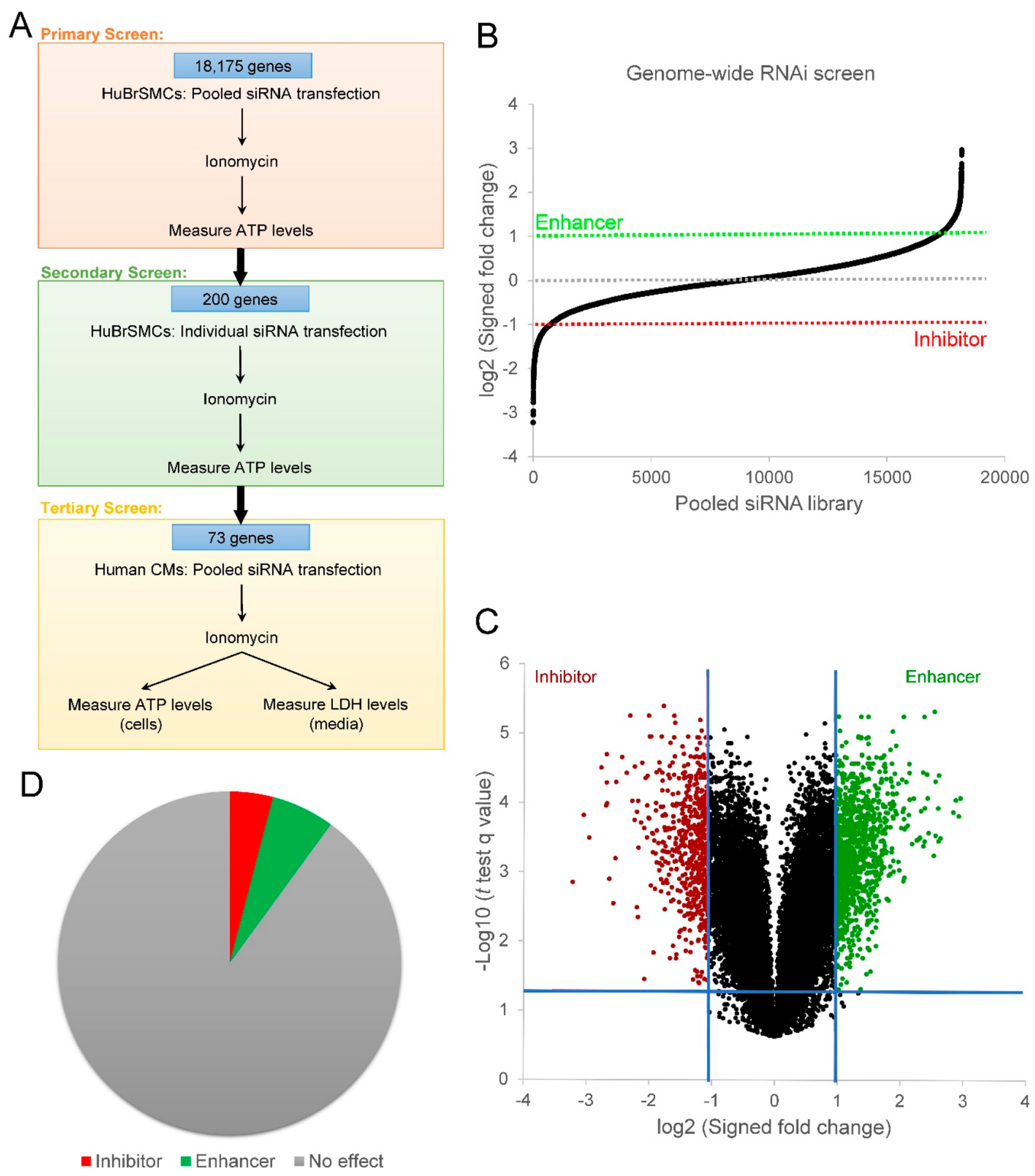

Figure 1. RNAi screen for regulators of necrosis. (A) Schematic diagram of the RNAi screen. (B) Scatter plot of log-transformed, nontargeting siRNA-normalized signed fold change of ATP levels in pooled siRNA-transfected cells. Data points are ordered from most negative (inhibitors) to most positive (enhancers). Dashed red or green lines are screen-specific cutoffs for inhibitors or enhancers, respectively. Dashed grey line indicates nontargeting control siRNAs. (C) Volcano plot of the $t$-test $q$-value (negative $\log _{10}$ ) against the signed fold change $\left(\log _{2}\right)$ from the primary screen. The horizontal blue line indicates the statistical significance level $(q=0.05)$, and the vertical blue lines represent a signed fold change of \pm 2 . Necrosis inhibitors (red dots, 1084) are defined as genes with a signed fold change less than -2 and $q<0.05$; necrosis enhancers (green dots, 743) are defined as genes with a signed fold change greater than 2 and $q<0.05$. (D) Pie chart showing the percentages of necrosis inhibitors and enhancers in the human genome based on data from the primary screen.

(3) Negative regulators of necrosis had a $\log _{2}$ (fold change) less than -1.5 . Therefore, the tertiary screen was performed against 73 genes (23 positive and 50 negative regulators of necrosis) using a pool of the top 2 siRNAs per gene. Human cardiomyocytes ( $80 \%$ confluent) were transfected in 96-well white-walled clear-bottomed plates with $25 \mathrm{nM}$ siRNA. Cells were incubated at $37{ }^{\circ} \mathrm{C} / 5 \% \mathrm{CO}_{2}$ for $72 \mathrm{~h}$ and then treated with ionomycin ( $15 \mu \mathrm{M}$, Cayman Chemical) for $4 \mathrm{~h}$ to induce necrosis. Cell viability was measured via CellTiter-Glo, as in the previous screens. Additionally, supernatant from each well was removed to separate 96-well plates for use with Cytotoxicity Detection Kit (LDH) (11644793001, Roche) 

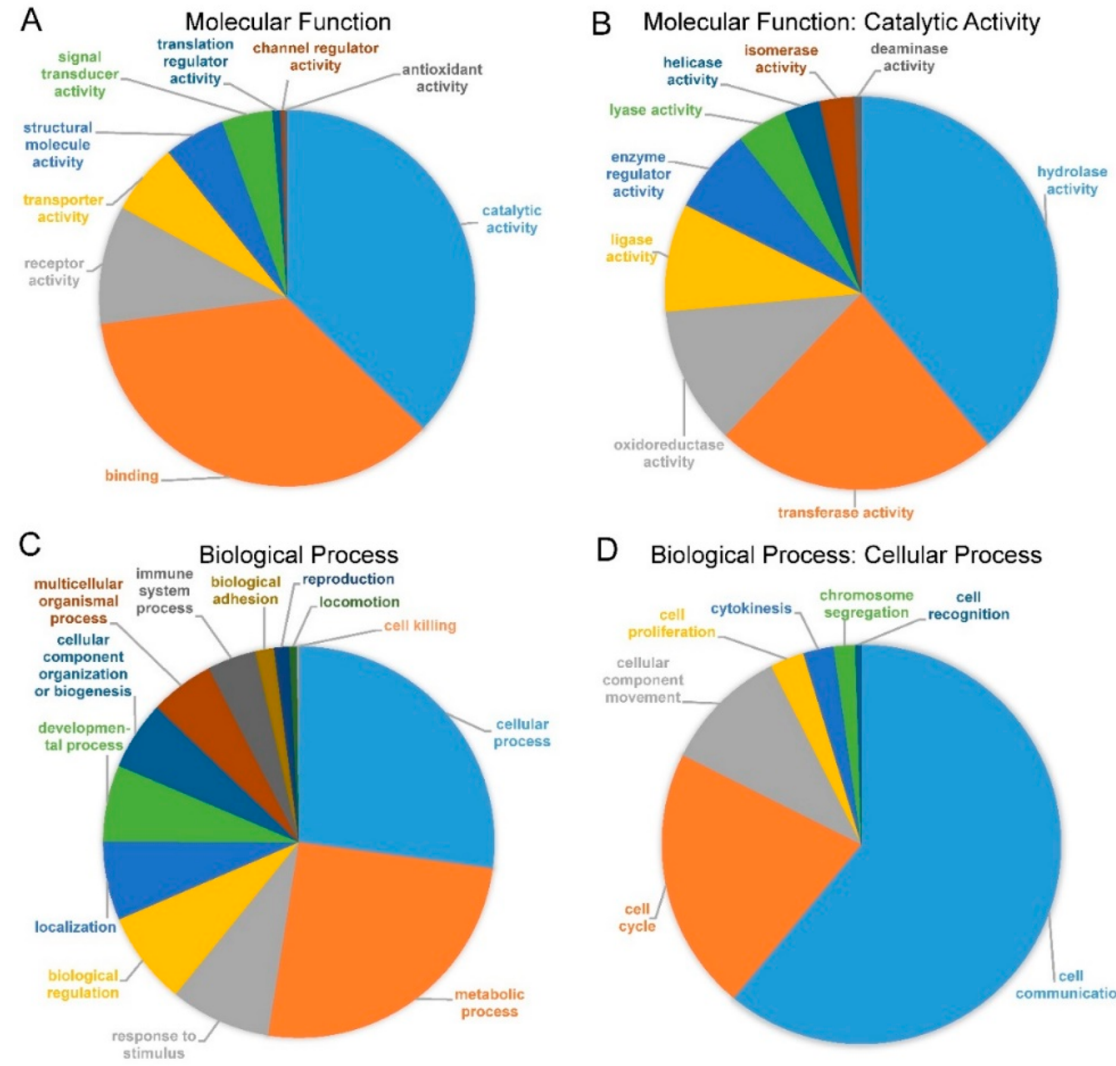

D Biological Process: Cellular Process
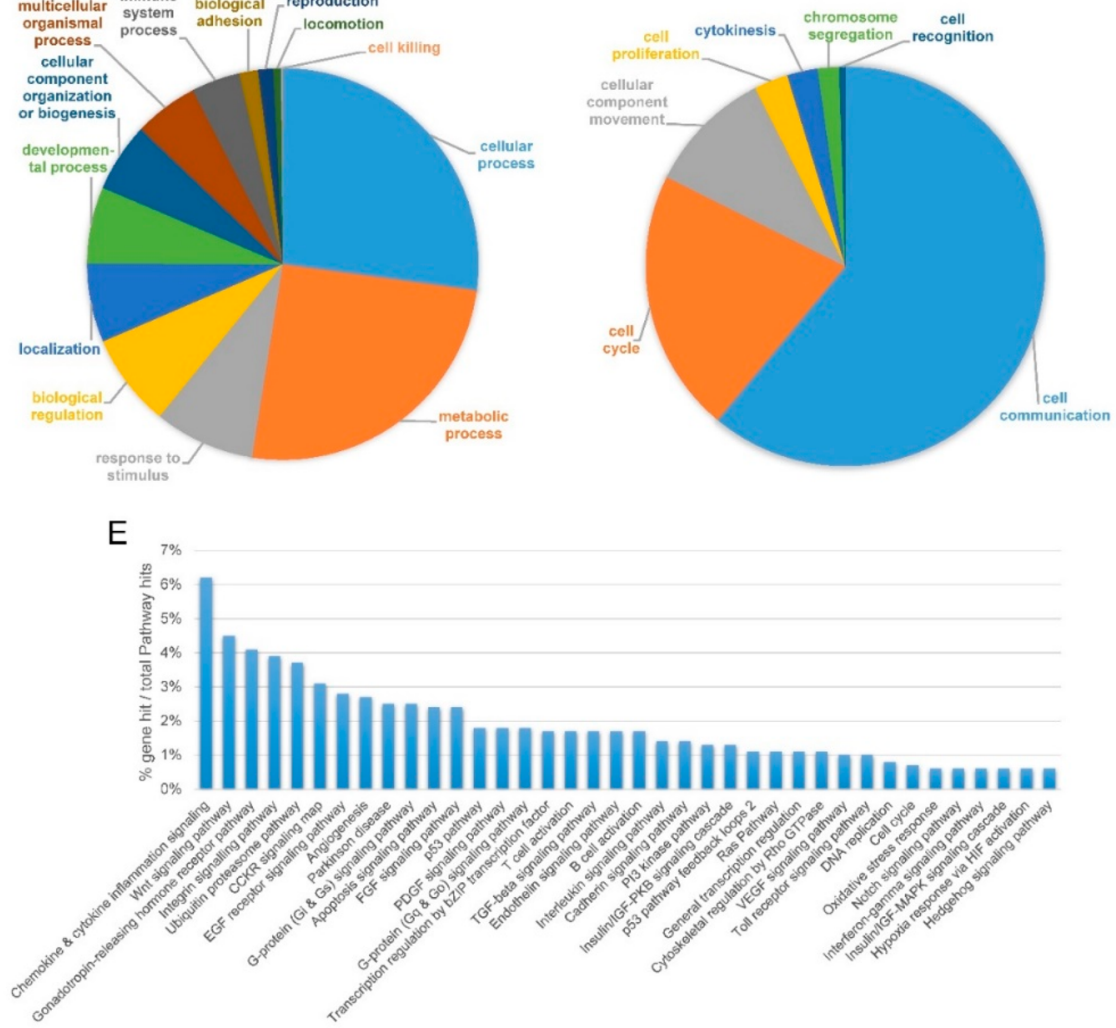

Figure 2. Enrichment of functional groups, biological processes and pathways in ionomycin-induced necrosis. (A) Candidate genes from the primary screen were grouped by molecular function using the PANTHER classification system. Top molecular functions related with necrosis were catalytic activity and binding. (B) Detailed protein categorization of the catalytic activity group in panel (A). Hydrolase and transferase activities were the primary catalytic activities involved in necrosis. (C) Candidate genes from the primary screen were grouped by biological process using the PANTHER classification system. More than half of all hits were associated with cellular and metabolic processes. (D) Detailed protein categorization of the cellular process group in panel (C). Cell communication and cell cycle were the predominant cellular processes identified. (E) Highly scored pathways in ionomycin-induced necrosis based on PANTHER pathway analysis.

according to the manufacturer's instructions. As before, nontargeting siRNAs and human UBB siRNAs (Dharmacon) served as negative and positive controls. For each run, CellTiter-Glo and LDH luminescence values were blanked against media alone. Sample means and standard errors were calculated; target means were normalized to nontargeting siRNA means. For each gene target, mean normalized values were calculated across all runs, and fold changes were calculated for ionomycin treatment versus vehicle only treatment. Error was propagated through all calculations according to appropriate formulas; one-sample two-tailed $t$ tests were performed with a null hypotheses of fold change equals 1. Significance was defined as $p<0.05$, and whiskers were plotted for means \pm standard errors.

Western Blotting. Cell lysates were loaded on to an SDSPAGE gel for electrophoresis. Proteins were then transferred onto a polyvinylidene fluoride (PVDF) membrane and incubated with the following antibodies: rabbit anti-PSMA6 (\#2459, Cell Signaling, 1:1000), rabbit anti-PSMB5 \#12919, Cell Signaling, 1:1000), rabbit anti-GAPDH (sc-25778, Santa Cruz, 1:1000), and mouse anti- $\alpha$-tubulin (T6074, Sigma, $1: 1000)$. 
A
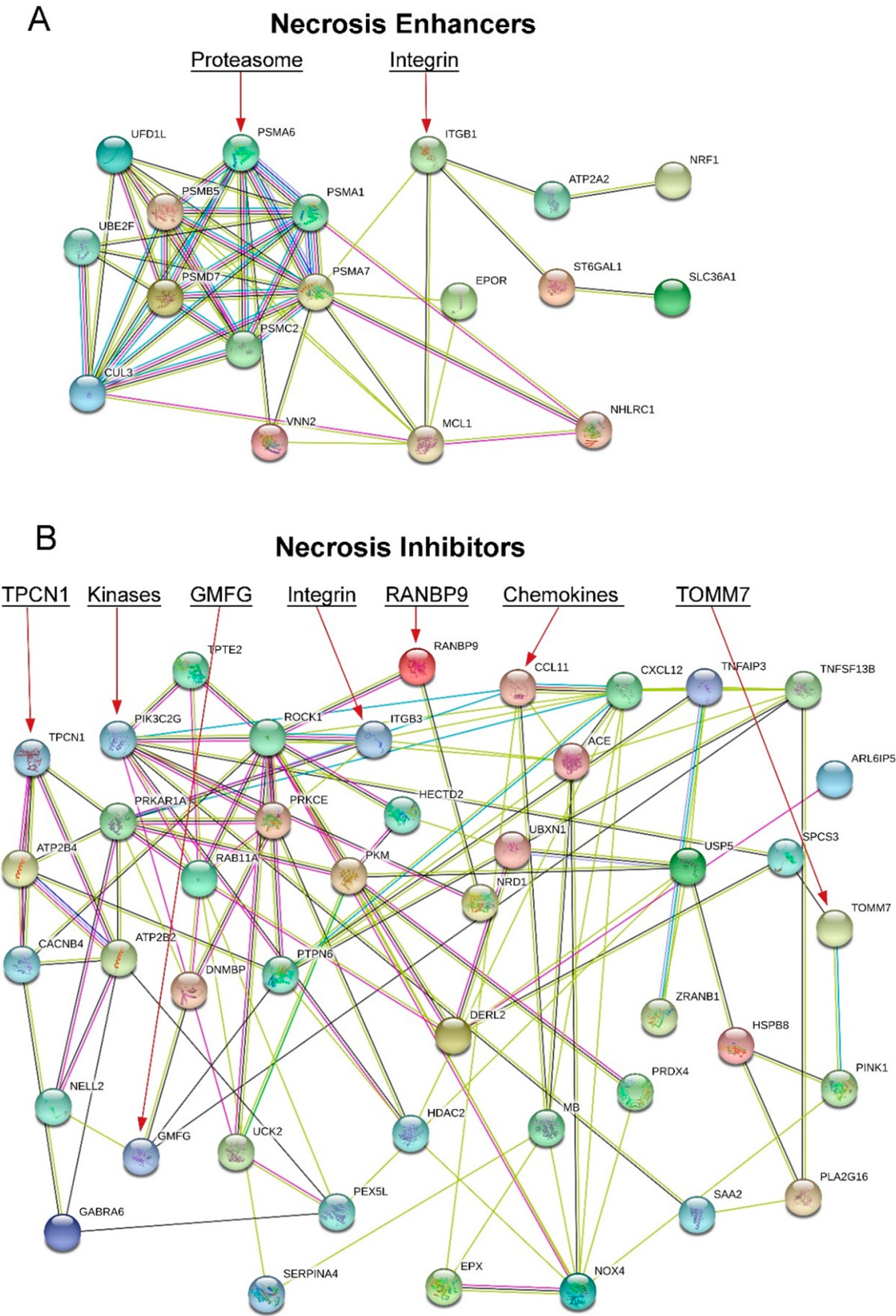

Figure 3. Protein interaction networks in ionomycin-induced necrosis. Potential protein-protein interactions between top candidates from the secondary screen were analyzed using the STRING program. (A) Necrosis enhancers; (B) necrosis inhibitors. Specific groups of genes discussed in the text were indicated by arrows. The different colors for proteins were used only as a visual aid.

\section{RESULTS AND DISCUSSION}

Genome-Wide siRNA Screen Identified Novel Genes Modulating lonomycin-Induced Necrosis. Necrosis is now considered a controllable cell death routine, and studies suggest that several distinct, but likely interconnected, signal transduction pathways are in place to trigger necrotic death. However, details are largely unknown regarding the precise pathways involved, particularly those downstream of calcium overload which is the major inducer of ischemia-induced cell death. The overarching goal of this study was to identify novel mediators of cardiomyocyte necrosis to aid in the future development of therapeutic strategies to mitigate cardiac injury. To this end, we developed an automated assay to perform a rigorous, unbiased genome-wide screen to identify modulators of necrosis induced by the effective $\mathrm{Ca}^{2+}$ ionophore, ionomycin, which acts in a fashion similar to that of MLKL to promote $\mathrm{Ca}^{2+}$ influx and is a well-established inducer of necrosis in a number of cell types. ${ }^{8,9}$

We utilized human smooth muscle cells as a surrogate muscle cell line for the primary and secondary screens and then validated these genes in a tertiary assay using human cardiomyocytes (Figure 1A). Smooth muscle cells were chosen due their relative ease of use and inexpensive application as cardiomyocytes can only be passaged a limited number of times and require specialized extracellular-matrix-coated plates. Moreover, smooth muscle cells share many features with cardiomyocytes including (but not limited to) (1) sarcoplasmic reticulum controlled $\mathrm{Ca}^{2+}$-dependent excitation-relaxation coupling, (2) a high mitochondrial density relative to 
nonmuscle cells, (3) a similar oxidative phosphorylation capacity of individual mitochondria, and (4) the need for basal mitochondrial activity to meet short-term energy demands. ${ }^{18,19}$

For the primary screen, we performed a two-condition triplicate analysis screen with the Dharmacon human genomic siRNA library that contains 72700 siRNAs targeting 18175 genes. In brief, human smooth cells (Celprogen, passage 10, 700 cells/well) were transfected with pooled gene-specific siRNAs (25 nM, 4 siRNAs/gene) and either negative control nontargeting siRNAs or siRNAs for human UBB (a gene essential for cell viability) which served as a positive control for transfection. The siRNA screen repeatedly showed an assay window of $>80 \%$ by comparing the cell viability in UBB and nontargeting siRNA transfected controls that were transfected in alternating rows of each plate (Figure S1A). At $96 \mathrm{~h}$ following transfection, cells were treated with vehicle or ionomycin $\left(\mathrm{IC}_{50}=5 \mu \mathrm{M}\right.$, Cayman Chemical; Figure S1B) for $4 \mathrm{~h}$ to induce necrosis and cells were subjected to a Cell Titer Glo assay to quantify cellular ATP.

To determine essential cellular signaling networks that were specific to ionomycin-induced death, we subjected this data set to SAMq and signed-fold change- statistical analyses that rank genes based on their effect size with ionomycin treatment relative to their effect without treatment. Using this approach we effectively filtered out genes that influenced basal cell viability as such genes would not likely be good therapeutic targets. This analysis revealed 1084 genes that significantly ( $p$ $<0.05$ ) enhanced ionomycin-induced necrosis with a signed fold change $>2$ and 743 hits that inhibited necrosis with a signed fold change $<2$ when compared with nontargeting siRNA-transfected controls (Figure 1B,C). Importantly, known necrosis regulators were identified along with numerous other novel genes that were even more efficacious necrosis enhancers or inhibitors respectively (Excel File S1). As shown in the Volcano plot in Figure 1C, 168 genes enhanced necrosis with a signed fold change $>3$ and 120 genes inhibited necrosis with a signed fold change $<3(q<0.05)$. Overall, the primary screen indicated that $6.0 \%$ of genes in the human genome have the potential to enhance necrosis and that $4.1 \%$ of human genes encode proteins that function as endogenous inhibitors of necrosis (Figure 1D).

Subjection of this very exciting initial data set to pathway enrichment analysis using Ingenuity Pathway Analysis (IPA, Qiagen, www.ingenuity.com), the STRING database (http:// string-db.org; KEGG pathways), and PANTHER revealed some expected and many unexpected findings (Figures 2 and 3; Tables 1 and 2). As expected, the highest number of genes segregated with known Cell Death and Survival pathways and the highest functional subcategory was cardiovascular toxicity with the top canonical pathway being protein ubiquitination. Hypoxia signaling in the cardiovascular system was also a top pathway, which suggests that functional overlap exists between ionomycin-induced necrosis and cell death induced by ischemic heart disease. Several genes involved in integrin signaling were also identified, which is consistent with reports that loss of the cysteine-protease cathepsin $S$ or overexpression of thrombospondin-4 proved effective in blunting skeletal muscle necrosis in dystrophic mice by mechanisms that involved increased dependence of plasma membrane stabilization on integrin-extracellular-matrix interaction. ${ }^{20}$

However, many novel genes were identified, and a breakdown of the catalytic activities involved in necrotic
Table 1. Functional Classification of Candidate Genes and Major Signaling Pathways Involved in Ionomycin-Induced Necrosis (Ingenuity Pathway Analysis)

\begin{tabular}{|c|c|c|c|}
\hline name & \multicolumn{2}{|r|}{$p$-value } & $\begin{array}{c}\text { no. } \\
\text { molecules }\end{array}$ \\
\hline \multicolumn{4}{|c|}{ Molecular and Cellular Functions } \\
\hline cell death and survival & \multicolumn{2}{|c|}{$4.81 \times 10^{-09}-1.12 \times 10^{-02}$} & 405 \\
\hline gene expression & \multicolumn{2}{|c|}{$6.50 \times 10^{-09}-1.04 \times 10^{-04}$} & 251 \\
\hline $\begin{array}{l}\text { cellular growth and } \\
\text { proliferation }\end{array}$ & \multicolumn{2}{|c|}{$8.41 \times 10^{-08}-1.16 \times 10^{-02}$} & 383 \\
\hline post-translational modification & \multicolumn{2}{|c|}{$2.54 \times 10^{-07}-8.83 \times 10^{-03}$} & 82 \\
\hline protein degradation & \multicolumn{2}{|c|}{$2.54 \times 10^{-07}-5.31 \times 10^{-06}$} & 65 \\
\hline \multicolumn{4}{|c|}{ Cardiovascular Toxicity } \\
\hline cardiac infarction & \multicolumn{2}{|c|}{$3.54 \times 10^{-04}-3.84 \times 10^{-01}$} & 26 \\
\hline heart failure & \multicolumn{2}{|c|}{$3.39 \times 10^{-03}-5.46 \times 10^{-01}$} & 26 \\
\hline cardiac pulmonary embolism & \multicolumn{2}{|c|}{$4.52 \times 10^{-02}-9.66 \times 10^{-02}$} & 4 \\
\hline $\begin{array}{l}\text { cardiac congestive cardiac } \\
\text { failure }\end{array}$ & \multicolumn{2}{|c|}{$5.70 \times 10^{-02}-5.70 \times 10^{-02}$} & 10 \\
\hline cardiac arrhythmia & \multicolumn{2}{|c|}{$9.15 \times 10^{-02}-5.89 \times 10^{-01}$} & 19 \\
\hline \multicolumn{4}{|c|}{ Top Canonical Pathways } \\
\hline \multicolumn{2}{|l|}{ name } & $p$-value & ratio \\
\hline \multicolumn{2}{|l|}{ protein ubiquitination pathway } & $8.09 \times 10^{-16}$ & $\begin{array}{l}68 / 254 \\
(0.268)\end{array}$ \\
\hline \multicolumn{2}{|l|}{ glucocorticoid receptor signaling } & $7.42 \times 10^{-06}$ & $\begin{array}{l}47 / 258 \\
\quad(0.182)\end{array}$ \\
\hline \multicolumn{2}{|c|}{$\begin{array}{l}\text { hypoxia signaling in the cardiovascular } \\
\text { system }\end{array}$} & $4.91 \times 10^{-05}$ & $17 / 63(0.27)$ \\
\hline \multicolumn{2}{|c|}{ PPARa/RXRa activation } & $1.41 \times 10^{-04}$ & $\begin{array}{l}31 / 165 \\
(0.188)\end{array}$ \\
\hline \multicolumn{2}{|l|}{ cAMP-mediated signaling } & $1.01 \times 10^{-03}$ & $\begin{array}{l}35 / 216 \\
(0.162)\end{array}$ \\
\hline
\end{tabular}

Table 2. Pathway Enrichment Analysis Using the STRING Database (KEGG Pathways)

\begin{tabular}{lcl}
\multicolumn{1}{c}{ pathway description } & $\begin{array}{c}\text { observed gene } \\
\text { count }\end{array}$ & $\begin{array}{c}\text { false discovery } \\
\text { rate }\end{array}$ \\
ubiquitin mediated proteolysis & 40 & $2.43 \times 10^{-10}$ \\
proteasome & 19 & $6.78 \times 10^{-08}$ \\
$\begin{array}{l}\text { neuroactive ligand-receptor } \\
\text { interaction }\end{array}$ & 50 & $1.11 \times 10^{-05}$ \\
Epstein-Barr virus infection & 37 & $9.85 \times 10^{-05}$ \\
viral carcinogenesis & 33 & 0.00136 \\
metabolic pathways & 137 & 0.00239 \\
NF- $\kappa$ B signaling pathway & 19 & 0.00523 \\
HTLV-I infection & 39 & 0.00594 \\
pathways in cancer & 45 & 0.017 \\
chronic myeloid leukemia & 15 & 0.017 \\
cyanoamino acid metabolism & 4 & 0.0357 \\
inositol phosphate metabolism & 13 & 0.0357 \\
type I diabetes mellitus & 10 & 0.0357
\end{tabular}

execution revealed that a remarkably diverse group of enzymes are involved including hydrolases, transferases, oxoreductases, ligases, lyases, and helicases (Figure 2). These findings indicate that a combinatorial inhibitor approach will likely be required to mitigate necrosis. Some surprises were also found when exploring protective genes, in that this list included an overrepresentation of genes involved in cell-cell communication (Figure S2).

We next performed a secondary validation screen in which we utilized four individual siRNAs to target 100 necrosis enhancers (signed fold change $>2$ ) and 100 necrosis inhibitors (signed fold change $<2$ ). Top-ranking necrosis-regulating genes from the secondary screen were identified by selecting 
those in which at least 2 out of the 4 siRNAs significantly altered cell survival $(q<0.05$ versus nontargeting siRNA). These criteria identified 23 necrosis enhancers and 50 necrosis inhibitors as validated targets (Excel File S2). To further analyze potential physical interactions and functional associations between the proteins encoded by these genes, we again ran these hits through the STRING database (http://string-db. org). As shown in Figure 3, the proteasome and cullin3 remained among the major protein complexes required for ionomycin-induced necrosis. Integrin family members integrin $\beta 1$ and $\beta 3$ appeared to play counteractive roles in necrosis, with $\beta 1$ being a necrosis enhancer and $\beta 3$ as well as the associated Rho-associated protein kinase 1 (ROCK1) being antinecrotic. Interestingly, chemokines CXCL12 (C-X-C motif chemokine ligand 12) and CCL11 (C-C motif chemokine 11) were also identified as necrosis inhibitors, suggesting that inhibition of necrosis may represent a novel mechanism underlying CXCL12/CXCR4-dependent cardioprotection. ${ }^{21}$ Finally, major protein kinase subunits PIK3C2G (phosphoinositide 3-kinase (PI3K) family kinase catalytic subunit), PRKCE (protein kinase $\mathrm{C}$ epsilon $(\mathrm{PKC} \varepsilon)$ ), and PRKAR1A (protein kinase A type 1a regulatory subunit) are also among the highly scored hits, indicating that PI3K, PKC, and PKA pathways may play a role in ionomycin-induced necrosis.

Screen for Necrosis Regulators in Human Cardiomyocytes. To aid in the identification of key regulators of cardiomyocyte necrosis, we performed a tertiary screen in cultured human cardiomyocytes (Cellprogen). Moreover, because permeabilization of the plasma membrane and release of intracellular enzymes such as lactate dehydrogenase (LDH) is a key signature for necrotic cells (but not apoptotic cells), we modified this tertiary screen to assess both intracellular ATP and extracellular LDH levels. We obtained a reliable $z$-score of 0.7 with an ionomycin $\mathrm{IC}_{50}$ of $15.5 \mu \mathrm{M}$ using Cell Titer Glo as a readout for ATP levels in these cells (data not shown). The higher resistance to ionomycin likely results from the remarkable ability of cardiomyocytes to maintain calcium homeostasis by sequestration into intracellular compartments (including t-tubules which are not present in smooth muscle cells) and/or by active transport across the plasma membrane. Importantly, 40 out of 50 of the protective genes identified in our secondary screen in human bronchial smooth muscle cells also protected human cardiomyocytes from ionomycinmediated ATP depletion, while 7 of the identified 23 necrosis enhancers exacerbated ionomycin-mediated ATP depletion in cardiomyocytes (Excel File S3). With respect to $\mathrm{LDH}$ release, 13 of the 23 necrosis enhancers and 30 of the 50 protective genes from secondary screen had a reciprocal effect on $\mathrm{LDH}$ release in cardiomyocytes (Excel File S3), though only 7 inhibitors and 3 enhancers reached statistical significance of $p$ $<0.05$ in this assay (Figure 4). While several modifiers of cardiac necrosis were identified using this work flow, known differences between cardiomyocytes and smooth muscle cells in mitochondrial abundance, proton leakage, and ROS production (among others) may have limited our ability to identify some relevant targets for cardiac necrosis in the present study. Indeed, while our systematic approach to perform the primary and secondary screens in smooth muscle cells followed by a tertiary screen in cardiomyocytes was designed to rule out putative false positives, false negatives are formally possible.

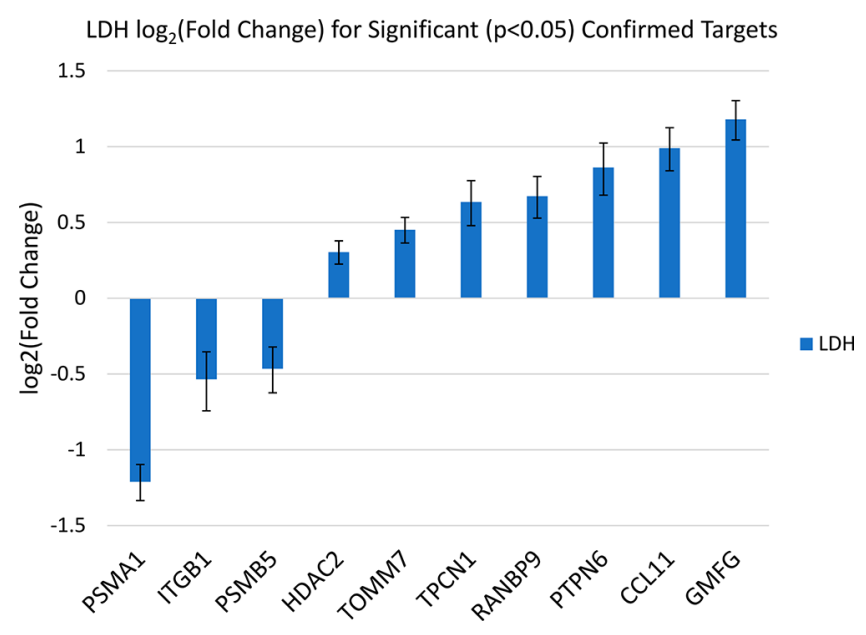

Figure 4. Tertiary RNAi screen data for regulators of cardiomyocyte necrosis as assessed by LDH. Bar graph of LDH $\log _{2}$ (fold change) for significant $(p<0.05)$ targets which recapitulated the secondary screen results.

One of the protective genes we identified in our tertiary screen as assessed by $\mathrm{LDH}$ release, the lysosomal calcium channel TPCN1, is a previously confirmed regulator of cardiomyocyte autophagy and cell death. Autophagy is an evolutionarily conserved intracellular degradation/recycling system that is fundamental for cellular homeostasis and metabolism ${ }^{22-24}$ and generally refers to the process by which organelles or long-lived proteins are engulfed by a doublemembraned autophagosome structure, which then fuses with lysosomes for degradation. Autophagy is enhanced following cardiac IR and inhibition of autophagy with bafilomycin A, which blocks lysosomal-mediated autophagosome degradation, worsens IR injury, and promotes the accumulation of damaged mitochondria under these conditions. ${ }^{31}$ TPCN1 functions to aid in autophagosome-lysosome fusion, and prior studies revealed that silencing of TPCN1 had no impact on cardiomyocyte viability under control culture conditions, but rather it significantly decreased the viability of cardiomyocytes exposed to ischemia or starvation. ${ }^{25}$ These studies are consistent with our findings and serve as validation of our approach to identify selective mediators of calcium-induced cardiomyocyte necrosis.

Another necrotic inhibitor we identified in our tertiary screen, TOMM7 (a component of the protein translocase of the outer mitochondrial membrane (TOM) complex), regulates a selective form of autophagy that removes damaged mitochondria referred to hereafter as mitophagy. Since mitochondria produce reactive oxygen species (ROS) as a byproduct of electron transport, even slight dysfunction can disrupt cellular redox balance leading to adverse lipid and protein modifications, the generation of toxic lipid intermediates, and mitochondrial DNA damage, all of which further perpetuate mitochondrial dysfunction and cell death. ${ }^{26,27}$ Although several mitochondrial quality control mechanisms have been described including mitochondrial fission and fusion, selective degradation of dysfunctional mitochondrial proteins, and mitochondrial biogenesis, ${ }^{28}$ mitophagy is thought to be the most important in nondividing cells such as cardiomyocytes and neurons. ${ }^{29,30}$ Several major cardiomyocyte mitophagy pathways have recently been described that involve accumulation of the serine/threonine kinase PINK1 on the 
outer membrane of damaged mitochondria, an event that promotes binding of the cytosolic E3 ubiquitin ligase, Parkin, followed by the subsequent ubiquitin-dependent recruitment of mitochondria into double-membraned autophagosomes. ${ }^{31-36}$ Interestingly, TOMM7 was originally identified in a screen for regulators of mitophagy and was subsequently shown to play a major role in the recruitment of Parkin to damaged mitochondria in neurons by promoting PINK1 stabilization. $^{37}$ While a functional role for TOMM7 in cardiomyocytes has not yet been reported, it is clear that Parkin has important cardioprotective functions. For example, depletion of Parkin or dynamin-related protein 1 (Drp1), a protein that inhibits mitochondrial fission and mitophagy, led to exacerbated injury following $\mathrm{IR}^{38,39}$ while the protective effects provided by ischemic preconditioning were abolished in Parkin KO mice. ${ }^{40}$ Thus, future studies on TOMM7 functions in the context of cardiac IR are warranted.

Additional genes that we identified as putative cardioprotective agents are consistent with their known functions. For example, we found that silencing of the Ran-binding protein, RanBP9, induced ionomycin-mediated cardiomyocyte necrosis. RanBP9 is highly expressed in the heart, but it has been primarily studied in cancer cells in which it has been shown to promote apoptosis. ${ }^{41}$ Mechanistically, RanBP9 downregulation promotes the expression of antiapoptotic $\mathrm{Bcl}-2$ family members $\mathrm{Bcl}-2$ and $\mathrm{Bcl}-\mathrm{X}_{\mathrm{L}}$ and, as a consequence, genotoxic-dependent recruitment of the pro-apoptotic family member Bax (which initiates activation of apoptosis by inducing mitochondrial permeabilization and cytochrome $\mathrm{c}$ release) is blunted in RanBP9-deficient cells. ${ }^{41}$ Since necrosis is often induced under conditions where apoptosis is blocked, ${ }^{42}$ these findings are consistent with our studies. However, they also indicate that RanBP9 targeting would not likely be therapeutically efficacious in the context of cardiac IR. Similarly, we found that HDAC2 depletion protected cells from ionomycin-mediated necrosis which is consistent with previous findings that HDAC2 is necessary for normal cardiac development. ${ }^{43}$ However, several groups have demonstrated that pan HDAC inhibitors can reduce infarct size and attenuate heart failure in preclinical animal models of $\mathrm{MI}$ and $\mathrm{IR}^{44}$ Taken together, our data support the idea that isoformselective HDAC inhibitors (which do not target HDAC2) may be more beneficial for the treatment of cardiovascular disease.

Two of the three top hits in our screen for cardioprotective agents are inflammatory mediators. CCL11 is a well-known cytokine, and PTPN6 has been shown to work in concert with the necrosis regulator RIPK1 to drive an NF- $\kappa \mathrm{B}$-mediated inflammatory signaling cascade in hematopoietic cells. ${ }^{45}$ While inflammation certainly plays a critical role in tissue damage following cardiac IR, the finding that these molecules provided protection to acute ionomycin-mediated cardiomyocyte death was somewhat surprising. However, it is known that mild activation of the innate immune axis can be protective against IR damage. ${ }^{46}$ Thus, it is formally possible that in our model, silencing of these genes attenuated a basal level of "preconditioning" prior to ionomycin treatment. It is also clear that cardiomyocytes secrete various cytokines which govern autocrine, paracrine, and endocrine functions including contractility, angiogenesis, local and systemic inflammation, and organismal metabolism. ${ }^{47-49}$ Of particular relevance to our findings, cardiomyocyte-restricted depletion of CXCR4 (the alpha chemokine receptor for the CCL11-related family member, CXCL12) exacerbated isoproterenol mediated cardiac injury in vivo, indicating a myocyte-autonomous protective role for this cytokine receptor. ${ }^{21}$ When taken together, these studies reveal the exciting possibility that CCL11 secretion may promote the establishment of an antinecrotic state among neighboring cardiomyocytes.

Finally, our top cardioprotective candidate, GMFG, is a novel antinecrotic target. This gene is a member of the ADF/ cofilin family which binds to $\operatorname{Arp} 2 / 3$ and serves to depolymerize actin. It has long been known that localized actin polymerization is necessary for mitochondrial fission and for mitochondrial movements. ${ }^{50}$ Interestingly, dephosphorylated cofilin is known to be targeted to mitochondria where it regulates DRP-1 mediated mitochondrial fission and mitophagy. ${ }^{51,52}$ While a direct role for GMFG in this process has not yet been shown, very recent findings that GMFG associates with the mitochondrial membrane ATPase ATAD $3 A,{ }^{53}$ which itself functions to mediate DRP-1-dependent mitochondrial fission, ${ }^{54}$ indicate that GMFG may also play a critical role in mitochondrial quality control. Our findings that GMFG depletion reciprocally regulated ATP and LDH levels in ionomycin-stimulated cardiomyocytes supports a role for this protein in regulating MTP-dependent necrosis (Figure 4 and Excel File S3).

Targeting the Proteasome for Cardioprotection. Because we were particularly interested in prioritizing druggable targets that could most likely impact human health, we chose to further validate the effect of targeting genes which served as executors of ionomycin-induced necrosis. To this end, we focused on the proteasome as we identified several subunits including PSMA1, 6, 7, and PSMB5 as enhancers of ionomycin-induced necrosis. To further validate these genes as targets for cardioprotection, we utilized isolated neonatal rat cardiomyocytes (NRVCMs) because they exhibit many of the characteristic features of their adult counterparts, and unlike the human cardiomyocyte cell line used for the tertiary screen, these cells do not divide in culture. ${ }^{15,55}$ We first focused on PSMB5 which encodes the proteasome $\beta 5$ subunit and contains the chymotrypsin-like active site. ${ }^{56}$ As shown in Figure 5A, transfection with siRNA utilized for our tertiary screen resulted in $>50 \%$ depletion of PSMB5 protein in NRVCMs. Importantly, PSMB5-depleted NRVCMs exhibited significantly increased cell viability (as measured by the MTT) and reduced $\mathrm{LDH}$ release following ionomycin treatment when compared to cells treated with control siRNA (Figure 5B). Similar results were obtained when the PSMA6 subunit was depleted in these cells (Figure S3).

To further corroborate our findings, we tested the possibility that bortezomib (BTZ), a reversible inhibitor that binds the proteasome $\beta 5$ subunit, would limit ionomycin-mediated necrosis. Indeed, as shown in Figure 5C, BTZ significantly attenuated $\mathrm{LDH}$ release and enhanced cell viability in ionomycin-treated NRVCMs. Similarly, previous studies showed that TNF-induced necroptosis was attenuated by carfilzomib, an irreversible proteasomal inhibitor that represses the chymotrypsin-like activity. ${ }^{57}$ Thus, while our study focused exclusively on calcium-mediated necrosis, the findings may be more broadly applicable. Indeed, previous studies in HeLa cells found that necrosis induced by calcium ionophore TNF $\alpha$ and ROS utilize overlapping mechanisms for necrosis execution. ${ }^{58}$ Importantly, previous studies indicated that the proteasome inhibitor PS-519 $9^{59,60}$ attenuated IR injury in preclinical mouse models. Likewise, proteasome inhibition with BTZ also protected against IR injury in skeletal muscle, ${ }^{61}$ peripheral 

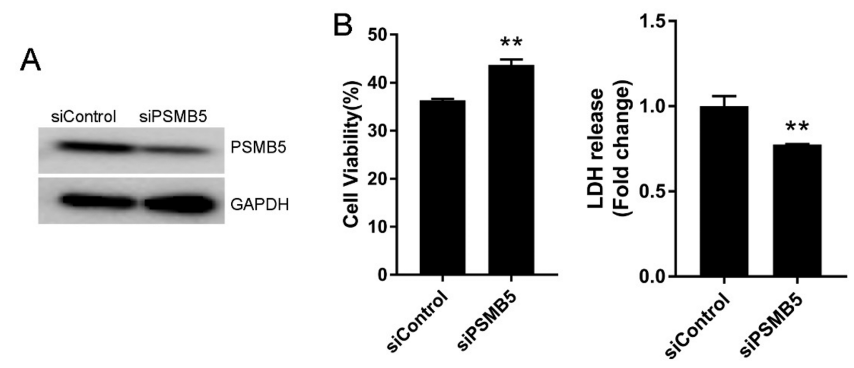

C
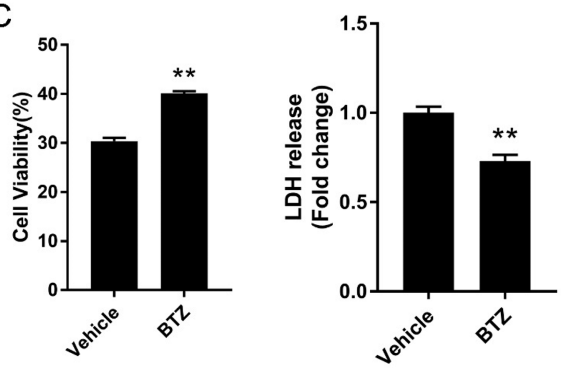

Figure 5. Proteasome inhibition suppresses ionomycin-induced necrosis in primary cardiomyocytes. (A) NRCMs were transfected with control (siControl) or PSMB5 siRNA (siPSMB5). Knockdown efficiency was assessed by Western blotting. (B) NRCMs transfected with siControl or siPSMB5 were incubated with ionomycin $(1 \mu \mathrm{M})$ for $1 \mathrm{~h}(n=3)$. Cell viability and LDH release were analyzed by MTT and LDH assays, respectively. ${ }^{* *}, p<0.01$ vs siControl. (C) NRCMs were pretreated with the proteasomal inhibitor bortezomib (BTZ, 1 $\mu \mathrm{M})$ for $1 \mathrm{~h}$ prior to incubation with ionomycin $(0.5 \mu \mathrm{M})$ for $1 \mathrm{~h}(n=$ 3). **, $p<0.01$ vs Vehicle control.

nerve, ${ }^{62}$ retina, $^{63}$ and steatotic liver, ${ }^{64}$ and proteasome inhibition with epoxomicin was associated with cardioprotection in hypertrophic cardiomyopathy. ${ }^{65}$ Collectively, these findings suggest that proteasome inhibition may provide protection from IR, at least in part, through repression of necrosis.

Cardiotoxicities are particularly prevalent in patients undergoing systemic therapy for malignancies, and in certain cases, this outcome results from drug-induced cardiotoxicity, particularly in patients who receive anthracycline-based treatments. Bortezomib is currently approved in the treatment of multiple myeloma and mantle cell lymphoma, ${ }^{56}$ and somewhat surprisingly, some case reports have described cardiac failure and other cardiotoxicities in treated patients. $^{66,67}$ However, a recent meta-analysis of data from 5718 patients failed to show significant cardiotoxicity with BTZ treatment. ${ }^{67}$ Thus, taken together with our findings and the preclinical studies described above, it is likely that the small subset of affected patients identified in these case studies may have suffered from pre-existing cardiovascular disease and/or elevated cardiovascular risk factors. Nonetheless, further studies are necessary to more fully understand the potential therapeutic value of bortezomib and related proteasomal inhibitors as cardioprotective agents.

\section{CONCLUSIONS}

We designed and applied an innovative automated, highthroughput, whole-genome siRNA discovery-based approach to identify human genes that specifically enhance or inhibit calcium overload-induced cardiomyocyte necrosis, a poorly understood area in which there is a pressing need to develop greater insight. Importantly, it is becoming increasingly clear that drug discovery and development are hampered by high attrition rates that are largely attributed to over reliance on model systems that do not adequately reflect processes in human organs. By starting with an unbiased screen in human muscle, then moving in the future to animal models for in vivo validation, we feel that our approach has a high likelihood of identifying clinically relevant targets for MI. It is noteworthy that human bronchial smooth muscle cells (HuBrSMCs) lack many characteristics of adult cardiomyocytes, such as the $t$ tubule system, mitochondrial abundance, terminal differentiation state, and so on. Therefore, it is possible that certain genes involved in necrosis may have not been picked in our screen. Nonetheless, this data set could prove useful for future risk ratio predictions as several genes we identified are current clinical targets for the treatment of non-CVD related pathologies and other disease-related polymorphisms. Finally, as many of these genes will likely have conserved functions in additional cell types, the future use of this knowledge could prove useful to attenuate excessive cell loss that accompanies muscular dystrophies or IR-mediated injury of the kidney, brain, or pancreas and thus have a broad and significant impact on human health.

\section{ASSOCIATED CONTENT}

\section{Supporting Information}

The Supporting Information is available free of charge on the ACS Publications website at DOI: 10.1021/acsptsci.9b00052.

Optimization of siRNA transfection and ionomycin treatment for the primary and secondary screens; classification and cellular localization of proteins crucial for ionomycin-induced necrosis; knockdown of PSMA6 suppresses ionomycin-induced necrosis in primary neonatal rat cardiomyocytes. (PDF)

Excel File S1: Primary screen data and analysis, including drug and control treatment values, calculated effect values, statistical test values, and GO analysis results (XLSX)

Excel File S2: Secondary screen data and analysis, including data for all individual siRNA targets (drug and control treatment values, calculated effect values, and statistical test values) (XLSX)

Excel File S3: Tertiary screen results, including CellTiter-Glo and $\mathrm{LDH}$ data (calculated effect values and statistical test values) (XLSX)

\section{AUTHOR INFORMATION}

\section{Corresponding Authors}

*501 Brinkhous-Bullitt Building, CB 7525, University of North Carolina, Chapel Hill, NC 27599. Tel.: (919) 843-5512. Fax: (919) 966-6718. E-mail: jmt3x@med.unc.edu (J.M.T.).

*PBS 423, 412 East Spokane Falls Boulevard, Spokane, WA 99202-2131. Tel.: (509) 358-7741. E-mail: zhaokang.cheng@ wsu.edu (Z.C.).

ORCID $\odot$

Joan M. Taylor: 0000-0002-8794-5167

\section{Notes}

The authors declare no competing financial interest.

\section{ACKNOWLEDGMENTS}

We thank Dr. Gary Johnson for providing valuable advice in assay development and access to the UNC siRNA screening 
facility, Brian Golitz (Director, UNC RNAi Screening Facility) for technical assistance in RNAi screen, Rachel Dee for assistance in statistical analysis, and Brian Mack for conception and development of the TOC graphic. This work was supported by $\mathrm{NIH} /$ National Heart, Lung, and Blood Institute grants HL-081844, HL-071054, and an American Heart Association grant $0355776 \mathrm{U}$ to J.M.T. Z.C. was supported by NIH Grant R00HL119605 and WSU College of Pharmacy.

\section{REFERENCES}

(1) Kung, G., Konstantinidis, K., and Kitsis, R. N. (2011) Programmed necrosis, not apoptosis, in the heart. Circ. Res. 108, 1017-36.

(2) Hartman, M. H. T., Groot, H. E., Leach, I. M., Karper, J. C., and van der Harst, P. (2018) Translational overview of cytokine inhibition in acute myocardial infarction and chronic heart failure. Trends Cardiovasc. Med. 28, 369-379.

(3) Vanden Berghe, T., Linkermann, A., Jouan-Lanhouet, S., Walczak, H., and Vandenabeele, P. (2014) Regulated necrosis: the expanding network of non-apoptotic cell death pathways. Nat. Rev. Mol. Cell Biol. 15, 135-147.

(4) Hitomi, J., Christofferson, D. E., Ng, A., Yao, J., Degterev, A., Xavier, R. J., and Yuan, J. (2008) Identification of a molecular signaling network that regulates a cellular necrotic cell death pathway. Cell 135, 1311-23.

(5) He, S., Wang, L., Miao, L., Wang, T., Du, F., Zhao, L., and Wang, X. (2009) Receptor interacting protein kinase-3 determines cellular necrotic response to TNF-alpha. Cell 137, 1100-11.

(6) Sun, L., Wang, H., Wang, Z., He, S., Chen, S., Liao, D., Wang, L., Yan, J., Liu, W., Lei, X., and Wang, X. (2012) Mixed lineage kinase domain-like protein mediates necrosis signaling downstream of RIP3 kinase. Cell 148, 213-27.

(7) Amin, P., Florez, M., Najafov, A., Pan, H., Geng, J., Ofengeim, D., Dziedzic, S. A., Wang, H., Barrett, V. J., Ito, Y., LaVoie, M. J., and Yuan, J. (2018) Regulation of a distinct activated RIPK1 intermediate bridging complex I and complex II in TNFalpha-mediated apoptosis. Proc. Natl. Acad. Sci. U. S. A. 115, E5944-E5953.

(8) Baines, C. P., Kaiser, R. A., Purcell, N. H., Blair, N. S., Osinska, H., Hambleton, M. A., Brunskill, E. W., Sayen, M. R., Gottlieb, R. A., Dorn, G. W., Robbins, J., and Molkentin, J. D. (2005) Loss of cyclophilin $\mathrm{D}$ reveals a critical role for mitochondrial permeability transition in cell death. Nature 434, 658-62.

(9) Nakagawa, T., Shimizu, S., Watanabe, T., Yamaguchi, O., Otsu, K., Yamagata, H., Inohara, H., Kubo, T., and Tsujimoto, Y. (2005) Cyclophilin D-dependent mitochondrial permeability transition regulates some necrotic but not apoptotic cell death. Nature 434, 652-8.

(10) Linkermann, A., Brasen, J. H., Darding, M., Jin, M. K., Sanz, A. B., Heller, J. O., De Zen, F., Weinlich, R., Ortiz, A., Walczak, H., Weinberg, J. M., Green, D. R., Kunzendorf, U., and Krautwald, S. (2013) Two independent pathways of regulated necrosis mediate ischemia-reperfusion injury. Proc. Natl. Acad. Sci. U. S. A. 110, 120249.

(11) Nakayama, H., Chen, X., Baines, C. P., Klevitsky, R., Zhang, X., Zhang, H., Jaleel, N., Chua, B. H., Hewett, T. E., Robbins, J., Houser, S. R., and Molkentin, J. D. (2007) Ca2+- and mitochondrialdependent cardiomyocyte necrosis as a primary mediator of heart failure. J. Clin. Invest. 117, 2431-44.

(12) Goonasekera, S. A., Lam, C. K., Millay, D. P., Sargent, M. A., Hajjar, R. J., Kranias, E. G., and Molkentin, J. D. (2011) Mitigation of muscular dystrophy in mice by SERCA overexpression in skeletal muscle. J. Clin. Invest. 121, 1044-52.

(13) Karch, J., Kwong, J. Q., Burr, A. R., Sargent, M. A., Elrod, J. W., Peixoto, P. M., Martinez-Caballero, S., Osinska, H., Cheng, E. H., Robbins, J., Kinnally, K. W., and Molkentin, J. D. (2013) Bax and Bak function as the outer membrane component of the mitochondrial permeability pore in regulating necrotic cell death in mice. eLife 2, No. e00772.
(14) Whelan, R. S., Konstantinidis, K., Wei, A. C., Chen, Y., Reyna, D. E., Jha, S., Yang, Y., Calvert, J. W., Lindsten, T., Thompson, C. B., Crow, M. T., Gavathiotis, E., Dorn, G. W., 2nd, O'Rourke, B., and Kitsis, R. N. (2012) Bax regulates primary necrosis through mitochondrial dynamics. Proc. Natl. Acad. Sci. U. S. A. 109, 6566-71.

(15) Cheng, Z., Dimichele, L. A., Rojas, M., Vaziri, C., Mack, C. P., and Taylor, J. M. (2014) Focal adhesion kinase antagonizes doxorubicin cardiotoxicity via p21. J. Mol. Cell. Cardiol. 67, 1-11.

(16) Moquin, D. M., and Chan, F. K. (2018) Loss-of-Function RNAi Screen to Identify Necrosis-Signaling Molecules. Methods Mol. Biol. 1857, 11-18.

(17) Tusher, V. G., Tibshirani, R., and Chu, G. (2001) Significance analysis of microarrays applied to the ionizing radiation response. Proc. Natl. Acad. Sci. U. S. A. 98, 5116-21.

(18) Chamley-Campbell, J., Campbell, G. R., and Ross, R. (1979) The smooth muscle cell in culture. Physiol. Rev. 59, 1-61.

(19) Park, S. Y., Gifford, J. R., Andtbacka, R. H., Trinity, J. D., Hyngstrom, J. R., Garten, R. S., Diakos, N. A., Ives, S. J., Dela, F., Larsen, S., Drakos, S., and Richardson, R. S. (2014) Cardiac, skeletal, and smooth muscle mitochondrial respiration: are all mitochondria created equal? Am. J. Physiol Heart Circ Physiol. 307, H346-52.

(20) Tjondrokoesoemo, A., Schips, T. G., Sargent, M. A., Vanhoutte, D., Kanisicak, O., Prasad, V., Lin, S. C., Maillet, M., and Molkentin, J. D. (2016) Cathepsin S Contributes to the Pathogenesis of Muscular Dystrophy in Mice. J. Biol. Chem. 291, 9920-8.

(21) Wang, E. R., Jarrah, A. A., Benard, L., Chen, J., Schwarzkopf, M., Hadri, L., and Tarzami, S. T. (2014) Deletion of CXCR4 in cardiomyocytes exacerbates cardiac dysfunction following isoproterenol administration. Gene Ther. 21, 496-506.

(22) Mizushima, N. (2007) Autophagy: process and function. Genes Dev. 21, 2861-73.

(23) Chiang, H. L., Terlecky, S. R., Plant, C. P., and Dice, J. F. (1989) A role for a 70-kilodalton heat shock protein in lysosomal degradation of intracellular proteins. Science 246, 382-5.

(24) Saftig, P., Beertsen, W., and Eskelinen, E. L. (2008) LAMP-2: a control step for phagosome and autophagosome maturation. Autophagy 4, 510-2.

(25) Garcia-Rua, V., Feijoo-Bandin, S., Rodriguez-Penas, D., Mosquera-Leal, A., Abu-Assi, E., Beiras, A., Maria Seoane, L., Lear, P., Parrington, J., Portoles, M., Rosello-Lleti, E., Rivera, M., Gualillo, O., Parra, V., Hill, J. A., Rothermel, B., Gonzalez-Juanatey, J. R., and Lago, F. (2016) Endolysosomal two-pore channels regulate autophagy in cardiomyocytes. J. Physiol. 594, 3061-77.

(26) Eisner, V., Csordas, G., and Hajnoczky, G. (2013) Interactions between sarco-endoplasmic reticulum and mitochondria in cardiac and skeletal muscle - pivotal roles in $\mathrm{Ca}(2)(+)$ and reactive oxygen species signaling. J. Cell Sci. 126, 2965-78.

(27) Song, M., Chen, Y., Gong, G., Murphy, E., Rabinovitch, P. S., and Dorn, G. W., 2nd (2014) Super-suppression of mitochondrial reactive oxygen species signaling impairs compensatory autophagy in primary mitophagic cardiomyopathy. Circ. Res. 115, 348-53.

(28) Sugiura, A., McLelland, G. L., Fon, E. A., and McBride, H. M. (2014) A new pathway for mitochondrial quality control: mitochondrial-derived vesicles. EMBO J. 33, 2142-56.

(29) Zhang, Y., Qi, H., Taylor, R., Xu, W., Liu, L. F., and Jin, S. (2007) The role of autophagy in mitochondria maintenance: characterization of mitochondrial functions in autophagy-deficient $S$. cerevisiae strains. Autophagy 3, 337-46.

(30) Twig, G., Elorza, A., Molina, A. J., Mohamed, H., Wikstrom, J. D., Walzer, G., Stiles, L., Haigh, S. E., Katz, S., Las, G., Alroy, J., Wu, M., Py, B. F., Yuan, J., Deeney, J. T., Corkey, B. E., and Shirihai, O. S. (2008) Fission and selective fusion govern mitochondrial segregation and elimination by autophagy. EMBO J. 27, 433-46.

(31) Deas, E., Plun-Favreau, H., Gandhi, S., Desmond, H., Kjaer, S., Loh, S. H., Renton, A. E., Harvey, R. J., Whitworth, A. J., Martins, L. M., Abramov, A. Y., and Wood, N. W. (2011) PINK1 cleavage at position A103 by the mitochondrial protease PARL. Hum. Mol. Genet. 20, 867-79. 
(32) Jin, S. M., Lazarou, M., Wang, C., Kane, L. A., Narendra, D. P., and Youle, R. J. (2010) Mitochondrial membrane potential regulates PINK1 import and proteolytic destabilization by PARL. J. Cell Biol. 191, 933-42.

(33) Youle, R. J., and Narendra, D. P. (2011) Mechanisms of mitophagy. Nat. Rev. Mol. Cell Biol. 12, 9-14.

(34) Chen, Y., and Dorn, G. W., 2nd (2013) PINK1-phosphorylated mitofusin 2 is a Parkin receptor for culling damaged mitochondria. Science 340, 471-5.

(35) Kane, L. A., Lazarou, M., Fogel, A. I., Li, Y., Yamano, K., Sarraf, S. A., Banerjee, S., and Youle, R. J. (2014) PINK1 phosphorylates ubiquitin to activate Parkin E3 ubiquitin ligase activity. J. Cell Biol. 205, 143-53.

(36) Koyano, F., Okatsu, K., Kosako, H., Tamura, Y., Go, E., Kimura, M., Kimura, Y., Tsuchiya, H., Yoshihara, H., Hirokawa, T., Endo, T., Fon, E. A., Trempe, J. F., Saeki, Y., Tanaka, K., and Matsuda, N. (2014) Ubiquitin is phosphorylated by PINK1 to activate parkin. Nature 510, 162-6.

(37) Hasson, S. A., Kane, L. A., Yamano, K., Huang, C. H., Sliter, D. A., Buehler, E., Wang, C., Heman-Ackah, S. M., Hessa, T., Guha, R., Martin, S. E., and Youle, R. J. (2013) High-content genome-wide RNAi screens identify regulators of parkin upstream of mitophagy. Nature 504, 291-5.

(38) Kubli, D. A., Zhang, X., Lee, Y., Hanna, R. A., Quinsay, M. N., Nguyen, C. K., Jimenez, R., Petrosyan, S., Murphy, A. N., and Gustafsson, A. B. (2013) Parkin protein deficiency exacerbates cardiac injury and reduces survival following myocardial infarction. J. Biol. Chem. 288, 915-26.

(39) Ikeda, Y., Shirakabe, A., Maejima, Y., Zhai, P., Sciarretta, S., Toli, J., Nomura, M., Mihara, K., Egashira, K., Ohishi, M., Abdellatif, M., and Sadoshima, J. (2015) Endogenous Drp1 mediates mitochondrial autophagy and protects the heart against energy stress. Circ. Res. 116, 264-78.

(40) Huang, C., Andres, A. M., Ratliff, E. P., Hernandez, G., Lee, P., and Gottlieb, R. A. (2011) Preconditioning involves selective mitophagy mediated by Parkin and p62/SQSTM1. PLoS One 6, No. e20975.

(41) Salemi, L. M., Maitland, M. E. R., McTavish, C. J., and SchildPoulter, C. (2017) Cell signalling pathway regulation by RanBPM: molecular insights and disease implications. Open Biol. 7, 170081.

(42) Nikoletopoulou, V., Markaki, M., Palikaras, K., and Tavernarakis, N. (2013) Crosstalk between apoptosis, necrosis and autophagy. Biochim. Biophys. Acta, Mol. Cell Res. 1833, 3448-3459.

(43) Montgomery, R. L., Davis, C. A., Potthoff, M. J., Haberland, M., Fielitz, J., Qi, X., Hill, J. A., Richardson, J. A., and Olson, E. N. (2007) Histone deacetylases 1 and 2 redundantly regulate cardiac morphogenesis, growth, and contractility. Genes Dev. 21, 1790-802.

(44) Weeks, K. L. (2019) HDAC inhibitors and cardioprotection: Homing in on a mechanism of action. EBioMedicine. 40, 21-22.

(45) Lukens, J. R., Vogel, P., Johnson, G. R., Kelliher, M. A., Iwakura, Y., Lamkanfi, M., and Kanneganti, T. D. (2013) RIP1-driven autoinflammation targets IL-1alpha independently of inflammasomes and RIP3. Nature 498, 224-7.

(46) Zuurbier, C. J., Abbate, A., Cabrera-Fuentes, H. A., Cohen, M. V., Collino, M., De Kleijn, D. P. V., Downey, J. M., Pagliaro, P., Preissner, K. T., Takahashi, M., and Davidson, S. M. (2019) Innate immunity as a target for acute cardioprotection. Cardiovasc. Res. 115, $1131-1142$

(47) Doroudgar, S., and Glembotski, C. C. (2011) The cardiokine story unfolds: ischemic stress-induced protein secretion in the heart. Trends Mol. Med. 17, 207-14.

(48) Pedersen, B. K., Akerstrom, T. C., Nielsen, A. R., and Fischer, C. P. (2007) Role of myokines in exercise and metabolism. J. Appl. Physiol. 103, 1093-8.

(49) Giudice, J., and Taylor, J. M. (2017) Muscle as a paracrine and endocrine organ. Curr. Opin. Pharmacol. 34, 49-55.

(50) Tilokani, L., Nagashima, S., Paupe, V., and Prudent, J. (2018) Mitochondrial dynamics: overview of molecular mechanisms. Essays Biochem. 62, 341-360.
(51) Rehklau, K., Hoffmann, L., Gurniak, C. B., Ott, M., Witke, W., Scorrano, L., Culmsee, C., and Rust, M. B. (2017) Cofilin1-dependent actin dynamics control DRP1-mediated mitochondrial fission. Cell Death Dis. 8, No. e3063.

(52) Li, G. B., Zhang, H. W., Fu, R. Q., Hu, X. Y., Liu, L., Li, Y. N., Liu, Y. X., Liu, X., Hu, J. J., Deng, Q., Luo, Q. S., Zhang, R., and Gao, N. (2018) Mitochondrial fission and mitophagy depend on cofilinmediated actin depolymerization activity at the mitochondrial fission site. Oncogene 37, 1485-1502.

(53) Aerbajinai, W., Ghosh, M. C., Liu, J., Kumkhaek, C., Zhu, J., Chin, K., Rouault, T. A., and Rodgers, G. P. (2019) Glia maturation factor-gamma regulates murine macrophage iron metabolism and M2 polarization through mitochondrial ROS. Blood advances. 3, 12111225.

(54) Zhao, Y., Sun, X., Hu, D., Prosdocimo, D. A., Hoppel, C., Jain, M. K., Ramachandran, R., and Qi, X. (2019) ATAD3A oligomerization causes neurodegeneration by coupling mitochondrial fragmentation and bioenergetics defects. Nat. Commun. 10, 1371.

(55) Li, F., Wang, X., Capasso, J. M., and Gerdes, A. M. (1996) Rapid transition of cardiac myocytes from hyperplasia to hypertrophy during postnatal development. J. Mol. Cell. Cardiol. 28, 1737-46.

(56) Goldberg, A. L. (2012) Development of proteasome inhibitors as research tools and cancer drugs. J. Cell Biol. 199, 583-8.

(57) Ali, M., and Mocarski, E. S. (2018) Proteasome inhibition blocks necroptosis by attenuating death complex aggregation. Cell Death Dis. 9, 346.

(58) Wang, Z., Jiang, H., Chen, S., Du, F., and Wang, X. (2012) The mitochondrial phosphatase PGAM5 functions at the convergence point of multiple necrotic death pathways. Cell 148, 228-43.

(59) Pye, J., Ardeshirpour, F., McCain, A., Bellinger, D. A., Merricks, E., Adams, J., Elliott, P. J., Pien, C., Fischer, T. H., Baldwin, A. S., Jr., and Nichols, T. C. (2003) Proteasome inhibition ablates activation of NF-kappa B in myocardial reperfusion and reduces reperfusion injury. Am. J. Physiol Heart Circ Physiol. 284, H919-26.

(60) Stansfield, W. E., Moss, N. C., Willis, M. S., Tang, R., and Selzman, C. H. (2007) Proteasome inhibition attenuates infarct size and preserves cardiac function in a murine model of myocardial ischemia-reperfusion injury. Ann. Thorac Surg. 84, 120-5.

(61) Park, J. W., Qi, W. N., Cai, Y., Urbaniak, J. R., and Chen, L. E. (2007) Proteasome inhibitor attenuates skeletal muscle reperfusion injury by blocking the pathway of nuclear factor-kappaB activation. Plast Reconstr Surg. 120, 1808-18.

(62) Park, J. W., Kim, K. M., Oh, K. J., Rhyu, I. J., and Jang, H. S. (2009) Proteasome inhibition promotes functional recovery after peripheral nerve reperfusion injury. J. Trauma 66, 743-8.

(63) Chen, F. T., Yang, C. M., and Yang, C. H. (2013) The protective effects of the proteasome inhibitor bortezomib (velcade) on ischemia-reperfusion injury in the rat retina. PLoS One 8, No. e64262.

(64) Tiriveedhi, V., Upadhya, G. A., Busch, R. A., Gunter, K. L., Dines, J. N., Knolhoff, B. L., Jia, J., Sarma, N. J., Ramachandran, S., Anderson, C. D., Mohanakumar, T., and Chapman, W. C. (2014) Protective role of bortezomib in steatotic liver ischemia/reperfusion injury through abrogation of MMP activation and YKL-40 expression. Transplant Immunol. 30, 93-8.

(65) Schlossarek, S., Singh, S. R., Geertz, B., Schulz, H., Reischmann, S., Hübner, N., and Carrier, L. (2014) Proteasome inhibition slightly improves cardiac function in mice with hypertrophic cardiomyopathy. Front. Physiol. 5, 484.

(66) Honton, B., Despas, F., Dumonteil, N., Rouvellat, C., Roussel, M., Carrie, D., Galinier, M., Montastruc, J. L., and Pathak, A. (2014) Bortezomib and heart failure: case-report and review of the French Pharmacovigilance database. Fundam. Clin. Pharmacol. 28, 349-52.

(67) Xiao, Y., Yin, J., Wei, J., and Shang, Z. (2014) Incidence and risk of cardiotoxicity associated with bortezomib in the treatment of cancer: a systematic review and meta-analysis. PLoS One 9, No. e87671. 